In: Stefan Hirschauer (Hg.): Un/doing Differences. Praktiken der Humandifferenzierung.

Weilerswist: Velbrück, S. 172-201.

\title{
Timo Heimerdinger
}

\section{Machen Trachten Menschenschläge? \\ Vestimentäre Praktiken alpiner Selbstessentialisierung}

\begin{abstract}
"Menschenschlag " - dieses Wort gehört, zumindest in kulturwissenschaftlichen Zusammenhängen, gegenwärtig ganz eindeutig zu den verba non grata, den gemiedenen, da konzeptionell unterentwickelten und daher mit dem Bann der Vorwissenschaftlichkeit belegten Begriffen. Zu nah rangiert dieser Begriff mit seiner Drift zur Ethnisierung an den epistemischen Abgründen der Essentialisierung oder der Naturalisierung, als dass er für eine wissenschaftliche Verwendung ernsthaft in Betracht käme. ${ }^{\mathrm{I}}$ Dabei geht es ja genau in einem Forschungszusammenhang, der Praktiken der Humandifferenzierung thematisiert, auch darum, zu untersuchen, wie Menschenschläge hergestellt und wahrgenommen werden. In eben diesem Sinne heben in einem Aufsatz aus dem Jahr I974 die beiden Tübinger Autoren Utz Jeggle und Gottfried Korff den Begriff sogar in den Titel: »Homo Zillertaliensis oder Wie ein Menschenschlag entsteht " (Jeggle \& Korff, I974a). Sie entwickeln in ihrem Text die These, dass der »Homo Zillertaliensis « als Produkt einer ökonomisch motivierten Selbstfolklorisierung verstanden werden müsse, als Resultat eines Wechselspiels aus Selbstdarstellung nach außen und dann ins Tal zurückwirkender Erwartungshaltung der Gäste: Die Touristen wollen »drinnen« im Tal genau jene singenden, jodelnden, pittoresk-urigen Tiroler wiederfinden, denen sie einst als Handschuh-Wanderhändler irgendwo »draußen « begegnet waren. Dieses Bild, so die Autoren, verfestigte sich zunehmend als Selbst- wie Fremdkonzept gleichermaßen. Sie interpretieren diesen Prozess vor dem Hintergrund einer »ökonomischen Notwendigkeit des Folklorismus « an anderer Stelle als »Entwicklung des Zillertaler Regionalcharakters « (Jeggle \& Korff, I974b) und schließen ihren Text mit dem Satz: »Das Trachtenkostüm ist zur Zwangsjacke geworden « (Jeggle \& Korff, ı974a, S. I 88). ${ }^{2}$

I Leimgruber (200I, S. I I I, FN 25) weist auf die Verwendung des Begriffes im Jahr 1937 durch Robert Ritter in einem sog. rassehygienischen Kontext hin, bei Kammerhofer-Aggermann (20I4) finden sich im Zusammenhang mit historischer Trachtenforschung Verwendungsbelege für die Begriffe » Menschentypen « und »Volkstypen «.

2 Dieses soziale Zwangsmoment betont auch Goffman, wenn er von Menschen als Gefängniswärtern ihrer selbst in den Zellen »anerkannter Eigenschaften «
\end{abstract}


Mit dieser kompakten Formulierung fassen sie eine zentrale Erkenntnis der volkskundlichen Folklorismusdebatte zusammen und bringen zudem auf den Punkt, was ihrer Meinung nach in diesem speziellen Zillertaler Fall der touristisch motivierten Induktion die Herstellung eines Menschenschlags durch trachtige Kostümierung auf der einen Seite von dem » Machen von Leuten durch Kleider « - durchaus im Sinne der Novelle Gottfried Kellers - auf der anderen Seite unterscheide: Es sei der Zwangscharakter, welcher Menschen hier zu Kollektiven zusammenbacke, insbesondere dann, wenn sich die Widersprüche der Selbstfolklorisierung zur Selbstverständlichkeit verfestigen und die Vergangenheit daher einfach nicht mehr vergehen will (vgl. Wietschorke, 20 I2). Derart zu Kollektiven gemachte Menschen sind in dieser Form nur dann längerfristig haltbar, wenn dieser Kohäsionseffekt sowohl nach innen wie nach außen, das heißt sowohl als Identifikations- wie als Distinktionsmechanismus wirksam wird, wie auch andere Beiträge in diesem Band aufzeigen (unter anderem Auer, Lentz, Scheiding).

Die Rolle von Kleidung bei solchen Prozessen ist Ausgangspunkt und Gegenstand dieses Textes: Meine Frage ist, ob und wie die Verwendung und Kontextualisierung von Trachten gegenwärtig in Tirol als Praxis der Herstellung von Differenzen und Differenzmarkierungen zwischen Menschen und Menschengruppen begriffen werden kann. Nach grundlegenden Ausführungen zum Zusammenhang von Kleidung und Gemeinschaftsbildung insgesamt (I) werden zunächst einige Informationen zum Hintergrund dieses Beitrags, einem Forschungsprojekt zum Zusammenhang von Nationalsozialismus und Trachtenpraxis in Tirol geboten (2). Im Anschluss geht es um unterschiedliche Formen der Differenzmarkierungen. Im Trachtenwesen selbst spielen diese bekanntlich eine zentrale Rolle, wenn etwa Ordnungen entlang der Kriterien Raum, Epoche, Festtag-Werktag, Geschlecht oder Familienstand als Bestandteile einer trachtenspezifischen Zeichensprache etabliert werden und zudem die Differenz zwischen Trachtlern und Nicht-Trachtlern implizit und explizit verhandelt wird (3). Weitaus weniger bekannt und offensichtlich ist jedoch, wie relevant und weitreichend Differenzmarkierungen im Inneren des Trachtenfeldes sind. So ist die Unterscheidung in echte Trachtler und nur trachtig gekleidete Personen (4) eine ebenso wichtige Frage wie das Qualitätsbewusstsein für die verwendeten Materialien (5). Zusammenfassend zeigt sich, dass die gegenwärtige Trachtenpraxis jenseits der in der Literatur bereits breit diskutierten Zuschreibungen von Regionalität und Traditionalität vor dem Hintergrund der in diesem Band verhandelten Ansätze insbesondere als ein Verfahren der menschlichen Selbstessentialisierung gedeutet werden kann (6).

schreibt, welche die Menschen jedoch gerne mögen (vgl. Goffman, I986, S. I 5). 


\section{Kleidung und Gemeinschaftsbildung}

Die Imagination von Menschenkollektiven, mithin die Bildung von Gruppen insgesamt, steht immer in der Dialektik von Differenzverstärkung nach außen und Differenzminimierung im Inneren - wenn auch in unterschiedlicher Intensität. Maßgeblich für die Wirksamkeit dieses Wechsel- und Zusammenspiels ist die Frage, ob dieser Doppelmechanismus sowohl für die Partizipierenden als auch für die $\mathrm{Zu}-$ schauenden in hinreichendem Maße wahrnehmbar und erlebbar wird. Die Prägnanz und Deutlichkeit der Unterscheidungsbildung macht hier den Unterschied. Viele kulturelle Praktiken spielen dabei eine Rolle: praktisch sämtliche Formen des Konsums, die habituelle Gebärdung in Sprache, Körper oder Auftreten. Der Bereich der Kleidung ist hierbei jedoch exponiert, weil Kleidung durch ihren ostentativen Charakter sowohl ein hohes Maß an Sichtbarkeit nach außen aufweist als auch zugleich als materiell-körpernahe Praxis mit haptisch-erfahrungsbezogener Dimension einen reflexiven Bezug zur Trägerin herstellt (Grigo, 20I 5, S. 4I-46 und 49-5I). Und dies gilt sowohl für die Beobachter wie die Akteure selbst, denn im Spiegel sieht der Mensch nicht nur seine Kleidung, sondern - zwangsläufig damit verbunden auch sich selbst in dieser Kleidung, während er sie haptisch-sensuell erfährt. Kleidung ist also ein besonders effizientes Mittel zur kulturellen Identitätsproduktion und Identitätskommunikation. Aus diesem unmittelbaren Wechselspiel von Selbst- und Fremdwahrnehmung auf sensorischer wie semiotischer Ebene folgt ein gesteigertes wissenschaftliches Interesse an dem Zusammenhang von Kleidung und Gemeinschaftsbildung. ${ }^{3}$ Dieser erweist sich als besonders stark und vital und stellt zugleich ein Parade-Anwendungsbeispiel für eine ebenso zeichen- wie praxistheoretisch informierte Kulturanalyse dar, die Kleidung als Mittel der Schaffung und Repräsentation sozialer Ordnungen untersucht. ${ }^{4}$

Phänomenologisch und funktional zu unterscheiden ist jedoch nach verschiedenen Kleidungsformen, wobei sich hinsichtlich der Kategorisierung jenseits der alltagssprachlichen Verwendung rasch erhebliche Probleme ergeben. So plausibel eine Unterscheidung zum Beispiel in Uniformen (Polizei oder Militär), Uniformierungen (Kittel für Pflegekräfte), Arbeitskleidung (blauer Anton oder blauer Anzug), jugendkulturelle Accessoires (Basecap) und religiöse Kleidung (Mönchskutte), Mode (Minirock, Norwegerpulli, Jeans) und Tracht (Schürze und

4 Vgl. etwa A. König, 2007, S. 45-50, wo im Anschluss an Bourdieu und Goffman Fragen der Habitustheorie mit denen der Selbstpräsentation zusammengeführt werden. 
Janker) auf den ersten Blick auch scheinen mag, so problematisch bis unmöglich ist sie spätestens auf den zweiten. Religiöse Kleidung kann Arbeitskleidung sein, Arbeitskleidung kann modisch werden und Mode als Uniformierung wirken.

Mit Blick auf das Thema dieses Textes: Auch der landläufig und in früherer volkskundlicher Forschung oft postulierte Gegensatz zwischen Mode (rascher Wandel) und Tracht (Traditionalität und Konstanz) wurde zwischenzeitlich grundlegend dahingehend relativiert, dass vermeintlich relativ stabile Trachtenformen vielfältige je zeitspezifischen Impulse aufgegriffen haben und sich daher weitaus dynamischer entwickelt haben als vielfach angenommen. Das vermeintlich gegensätzliche Begriffspaar Mode und Tracht ist zur trennscharfen Gegenstandsbezeichnung untauglich und auch als analytische wissenschaftliche Begrifflichkeit längst obsolet, denn Tracht ist immer wieder sowohl zeitspezifisch beeinflusst als auch selbst zum Anlass für aktuelle Kreationen gemacht worden (Brückner, 1987). In der jüngeren Literatur wird »Tracht « daher viel eher als Wissensmodus oder »Denkstil « (Keller-Drescher, 20I 5) gesehen, als Wahrnehmungs- und Deutungsmuster also, Trachten sind als erfundene Traditionen und nur vermeintlich einheitliche und althergebrachte Kleidung analysiert worden (Keller-Drescher, 2015, S. I70). Dies ist in der Perspektive einer historisch informierten kulturwissenschaftlichen Kleidungsforschung evident, aber der subjektiven Realität und Realität stiftenden Wirkkraft dieses Denkstils im Bewusstsein vieler trachtenaffiner Akteurinnen samt Publikum tut dies keinen Abbruch. Denn dort halten sich offenbar weiterhin Vorstellungen von Traditionalität, Stabilität und eindeutiger regionaler Verortbarkeit, wenn von Tracht als dem »Kleid der Väter " die Rede ist. ${ }^{5}$

Eines jedenfalls lässt sich für Kleidung allgemein mit Fug und Recht behaupten: Sie spielt für Prozesse der Gemeinschaftsbildung eine wichtige Rolle, wenn auch in zu differenzierender Art und Weise. Uniformen vereinheitlichen, hierarchisieren und rücken den Rang vor das Individuum. Uniformierungen wie etwa einheitliche Kleidung in Firmen setzen jene ins Bild und machen die Trägerinnen zu Repräsentantinnen ihrer Arbeitgeber, Arbeitskleidung weist funktionale Bedeutung zu, und Kleidungsmode individualisiert und kategorisiert zugleich nach Aspekten wie Ökonomie, Modernität, Geschmack oder Modebewusstsein. Auch Trachten beziehungsweise jene Kleidungsstücke, die als solche bezeichnet und wahrgenommen werden, schaffen auf eine spezifische Art und Weise Gemeinschaft, nämlich durch die Behauptung der bewussten und dezidierten Eingliederung der Trägerin in eine diachrone Zeitdimension, die gewöhnlich mit dem Begriff der Tradition angesprochen wird.

5 So Oswald Gredler, der Obmann des Tiroler Landestrachtenverbandes, vgl. Darnhofer/Rapp 20I 5: 5. 
Mit dieser Behauptung von Einordnung in eine Praxis der Kontinuität und Dauer ist oft jene der räumlichen Verortung in Bezug auf eine bestimmte Region, einen Landstrich oder eine Ortschaft verbunden. Die Praxis- und Diskursform Tracht zeichnet sich also hinsichtlich der Gemeinschaftsbildung durch eine sowohl räumliche wie zeitliche Verankerungsbehauptung aus, die auf Vereinsebene auch noch durch eine soziale ergänzt werden kann. Dieser mehrfache Verankerungsgestus beansprucht damit, Konstituens eines starken räumlichen und zugleich über den Moment hinausreichenden, daher ungleichzeitigen Vergemeinschaftungseffekts zu sein.

Tracht produziert hier soziale Zugehörigkeit und Vereindeutigung und kann zudem als performativer Akt der Selbstessentialisierung gelesen werden: Die mit einer bestimmten Trachtenkleidung erfolgende räumliche Zuordnung unterliegt sozialen Regulationsmechanismen und erfolgt im Modus der Naturalisierung.

Nicht jede Person kann - auch nach längerer Wohndauer an einem bestimmten Ort - einfach so Legitimität für das Tragen der je ortsspezifisch als typisch angesehenen Trachtenkleidung beanspruchen. Hierfür ist das Placet der als einschlägig akzeptierten Elite notwendig. ${ }^{6}$ Augenscheinlich wird dies in dem bei Trachtenträgerinnen häufig anzutreffenden Sprachbild der »Erdung «, der »Verwurzelung « beziehungsweise des angeblichen Auffindens oder Erlebens von »Wurzeln ", welches mit dem Tragen von Tracht in Beziehung gesetzt wird: "Man fühlt sich geerdeter darin $« .7$ Mit dieser pflanzlichen Metaphorik geht nicht nur ein Moment der Selbstorganologisierung einher, sondern insbesondere funktional auch die Anrufung von Stabilität, Selbstevidenz und Natürlichkeit. In der Praxis des Trachttragens manifestieren und artikulieren sich diachrone und synchrone Kongruenz- und Gemeinschaftsbehauptungen: die mit »den Vätern «, die mit anderen Trachtenträger/innen und jene mit der örtlichen Bezugsgruppe an Gesinnungs-

6 Empirisch zeigte sich dies jüngst bei der ethnografischen Untersuchung eines Trachtennähkurses in Tirol. Im Kontext dieses Kurses bestimmte die Kursleiterin, welche Teilnehmerin in Abhängigkeit von Herkunft und Wohnort welches Kleid nähen »durfte«. Die teilnehmend-beobachtende Ethnografin wurde so auf das Muster »Obere Unterinntaler Tracht « verpflichtet, obwohl ihr eigentlich das Modell »Ötztaler Tracht « wesentlich besser gefallen hätte (Neuner-Schatz, 20I6, S. I 25). Es zeigt sich hier im Kurskontext ein Moment der Verweigerung der Anerkennung von Kontingenz, das Auer in diesem Band auch in soziolinguistischen Zusammenhängen beobachtet.

7 Obmann Oswald Gredler (Darnhofer \& Rapp, 201 5, S. 3). Zur Wurzelmetaphorik in der Kulturwissenschaft und den damit verbundenen Denkfiguren von Linearität und Monokausalität in soziobiologischer Akzentuierung vgl. theoretisch Köstlin, I999 und empirisch mit Blick auf gegenwärtige Trachtenpraktiken Neuner-Schatz, 2016, S. I3 I-I33. 
und Kleidungsgenossen. Die Idee und Empfindung des umfassenden Gehaltenseins in raumzeitlicher und sozialer Hinsicht leistet mit ihrem Plausibilitätsanspruch für die Trachtenträgerinnen entweder emotionale Beherbergung oder stellt eine Struktur des Zwanges dar - je nach Perspektive.

Damit zurück zum Homo Zillertaliensis: Dort geht es also nicht nur um die rein zeichenhafte und symbolische Herstellung von Kollektiven oder Identitäten, sondern vielmehr darum, dass Selbst- und Fremdbilder mit weit reichenden Konsequenzen amalgamiert und habitualisiert werden und auf diese Weise fast schon einen sozialontologischen Status bekommen, wie die Autoren für »den Zillertaler « speziell unter den Bedingungen einer stark entwickelten Tourismusindustrie ausführen: "Sein Verhalten, das er in Kontakten nach außen gelernt hat, ist ihm zur zweiten Natur geworden. Das Klischee, das er als Verkaufsstrategie zum besseren Absatz seiner 'heimischen Waren entwickelt hat, ist zum lebendigen Menschen geworden. Zwanghaft muß er sich auch zu Hause so geben, wie er in der Fremde war - er muß das nach außen aufgebaute Stereotyp verinnerlichen, er muß den Rollenerwartungen genügen, die der Fremde an ihn heranträgt « (Jeggle \& Korff, I974b, S. 47).

In diesem Prozess der kollektiven Internalisierung einer Rollenerwartung und damit der Herstellung eines Menschenschlages spielen, Jeggle und Korff deuten es an, neben diversen ökonomischen Bedingungen und Zwängen auch kulturelle Objektivationen wie Lieder, Dialekt, Sprachund Verhaltensformen (etwa das standardmäßige Duzen von Fremden) und nicht zuletzt auch habitualisierte Kleidungspraktiken, hier: die Verwendung von Trachten, eine wichtige Rolle. ${ }^{8}$

\section{Tiroler Trachtenpraxis: historische und gegenwärtige Verflechtungen}

Der Kontext, in dem dieser Text steht, ist ein aktuelles Innsbrucker Forschungsprojekt »Tiroler Trachtenpraxis im 20. und 2I. Jahrhundert ", das sich vornehmlich mit den Umtrieben, Vor- und Wirkungsgeschichten der nationalsozialistisch motivierten »Mittelstelle Deutsche Tracht« (1939-45) am Tiroler Volkskunstmuseum in Innsbruck be-

8 Dieser bei Jeggle und Korff nur en passant benannte Befund wurde von Hupfauf, 2016 bestätigt. Auch wenn es in dieser ethnomusikologischen Arbeit primär um die Verbreitung von Liedern geht, so wird anhand der Abbildungen (S. I2, I9, 20, I79) und auch im Text (S. IO, I4, 36, 74-76, I35) deutlich, dass die »trachtenähnlichen Kostüme « hierbei stets eine wichtige Rolle gespielt haben. 
fasst. ${ }^{9}$ Im Zentrum des Forschungsprojektes steht des Wirken von Gertrud Pesendorfer (I 895-I982), einer - wenn man so will - Innsbrucker Trachtenaktivistin, die als begabte Enthusiastin und später überzeugte Nationalsozialistin am Tiroler Volkskunstmuseum, ab 1927 zunächst als Sekretärin und dann ab 1939 als Leiterin der im Museum angesiedelten »Mittelstelle Deutsche Tracht ", einer Dienststelle der Reichsfrauenführung, steil Karriere machte. ${ }^{10}$ Ihre Aufgabe und Leidenschaft war die so genannte "Trachtenerneuerung ", eine Tätigkeit im Schnittfeld von Erforschung und Gestaltung des Trachtenwesens. Bestehende Trachtenmuster wurden feldforschend erhoben und auf dieser Basis wurden neue, zeitgemäßere, das heißt leichtere und alltäglich besser tragbare Trachten entworfen und auch in Nähkursen, in Publikationen und auf anderen öffentlichkeitswirksamen Wegen wie Vorträgen, Beratungen etc. popularisiert. Auch nach ihrer Absetzung als Museumschefin im Jahr I945 wurde Pesendorfer weiterhin in Tirol sehr wertgeschätzt, fand Arbeit und Aufgabe als Nähkursleiterin im Auftrag der Landwirtschaftskammer. Sie war als Trachtenexpertin und -beraterin weiterhin gefragt und publizierte vor allen Dingen mit großem Zeitverzug und mindestens ebenso großem Erfolg im Jahr I966 die tirolspe-

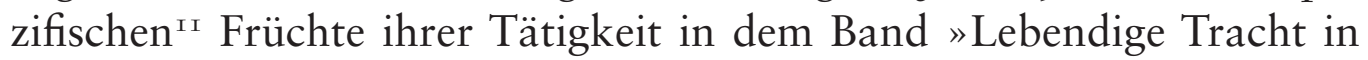
Tirol « (Pesendorfer, I966), der rasch zu einem Standardwerk für Tiroler Trachtenfragen avancierte, unschwer erkennbar an seiner Neuauflage im Jahr 1982. Sein Vertrieb seitens des Verlags wurde erst im Jahr 2013 im Kontext jener politischen Diskussionen (»Ploner-Debatte «) eingestellt, welche schließlich auch die Konzeption und öffentliche Finanzierung unseres Forschungsprojektes zu Folge hatten. Hierbei geht es neben einer historischen Aufarbeitung auch, wenn auch nach- oder beigeordnet, um eine Untersuchung der Spät- und Langzeitwirkungen der Pesendorferschen Aktivitäten. ${ }^{\text {I2 }}$

Die Gesamtwirkung Pesendorfers für das Tiroler Trachtenwesen war hoch. Auch wenn sie nicht die Erfinderin des Konzepts von Talschaftstrachten an sich war, so hat sie einer systematischen Kategorisierung und Festschreibung von Trachtenmustern als les- und kommunizierbare

$9 \mathrm{Zu}$ Projektgenese und Zielsetzung vgl. Bodner \& Heimerdinger, 2016 und auch die Projekthomepage https://www.uibk.ac.at/geschichte-ethnologie/ee/ trachten.html. Ich danke Reinhard Bodner für Diskussionen und Rechercheunterstützung bei der Arbeit an diesem Text!

Io Vgl. hierzu grundlegend wenn auch streckenweise herablassend-gönnerhaft oder polemisch Wallnöfer, 2008.

I I Inklusive Südtirol, ein Umstand, der damals wie heute eine gewisse politische Ladung mit sich bringt.

I 2 Zudem steht das Projekt auch im Kontext der jüngeren fachlichen Auseinandersetzung mit Begriff, Konzept und Praxis von »Volkskultur« (vgl. u.a. Wietschorke \& Schmidt-Lauber, 20I6; Eggmann \& Oehme-Jüngling, 20I3). 
Zeichensprache erheblichen Vorschub verschafft. Denn schon innerhalb des Trachtenwesens selbst spielen Differenzmarkierungen eine zentrale Rolle; sie erfolgen unter anderem nach den Kriterien Raum (Regionen und Talschaften), Epoche, Festtag-Werktag, Geschlecht (strikt heteronormativ) oder Familienstand. Solche Chiffrierungen und Ordnungsstrukturen sind nicht nur für das Trachtenwesen selbst konstitutiv, sondern auch als Wissensbestände für die Akteure ein wichtiger Bestandteil der internen Kommunikation und Vergemeinschaftung sowie der Bestimmung der internen Hierarchie im Rahmen von Fachsimpeleien und Wissenskonkurrenzen, auf die unten noch näher eingegangen werden wird: Wer weiß wie gut Bescheid und kann sich entsprechend legitimieren?

Dass es jedoch auch insgesamt zwischen »Tirol « und »Tracht « einen engen, geradezu innigen Konnex gibt, darauf verweist nicht nur die jahrzehntelange Popularität des Bandes »Lebendige Tracht in Tirol «, sondern dies hat sich auch allgemein zu einer stereotypen Vorstellung über das Land und seine Leute verfestigt. Dieses Vorurteil der Trachtenaffinität bezieht sich nicht allein auf Tirol, sondern auf viele alpine Gegenden und nicht zuletzt auf Österreich insgesamt, wie es etwa im langen Wahlkampf um das Amt des Bundespräsidenten im Jahr 2016 wieder deutlich wurde. Auf unterschiedlichen Ebenen erfährt es kontinuierliche mediale Reaktualisierungen.

Für Tirol besonders prominent wie wirkmächtig war hierfür die vierteilige Erfolgsfernsehserie »Die Piefke-Saga « des Autors Felix Mitterer Anfang der I990er Jahre: eine weiterhin ebenso fest wie vital im kollektiven Gedächtnis präsente satirisch-genüssliche Thematisierung des Verhältnisses zwischen Tiroler Gastgebern und deutschen Gästen, personifiziert in der Berliner Familie Sattmann und der Tiroler Gastgeberfamilie Wechselberger - samt allen Klischees. Auf der einen Seite die vor Geld strotzenden, besserwisserischen und reichlich überheblichen Sattmanns, auf der anderen Seite die raffinierten, geldgierigen und durchtriebenen Tiroler Gastgeber. Beide Seiten sind sympathisch und unsympathisch zugleich, bekommen reichlich ihr Fett ab - eine lustig-böse Parade wechselseitiger Irrungen und Wirrungen, ein gemeinsames Wechselberger-Sattmann-Baby inclusive. Zumindest gilt dies für die Teile I bis 3. Im vierten Teil der Saga kippt die Darstellung ins grotesk-dystopische, ein apokalyptisch-futuristisches Szenario irritierte seinerzeit die Zuschauer: Tirol ist hier in einer imaginierten nahen Zukunft nur noch eine auf Müllbergen errichtete Kunststoffkulisse, dank Technik gibt es gleichzeitig Sommer- und Wintertourismus, die Einheimischen wurden von japanischen Wissenschaftlern zu dauerfreundlichen und dauerjodelnden Dauergastgeber/innen umoperiert - und sie tragen allesamt Tracht, dabei ständig die Rede von der Rückbesinnung auf die Werte der Väter auf den Lippen. 
Bemerkenswert ambivalent sind die aktuellen Botschaften der Tiroler Tourismuswerbung in ihrem Blog. Während teilweise unverhohlen »Noch heute ist Tracht und Tirol untrennbar verbunden ${ }^{13}$ proklamiert wird, wird an anderer Stelle die Vorstellung, alle Tiroler »rennen nur in Trachten rum « als zwar populäres aber unzutreffendes Vorurteil zurückgewiesen, allerdings auf der Bildebene in Form einer pseudodokumentarischen Werbefotografie zugleich wieder aufgerufen: ${ }^{14}$

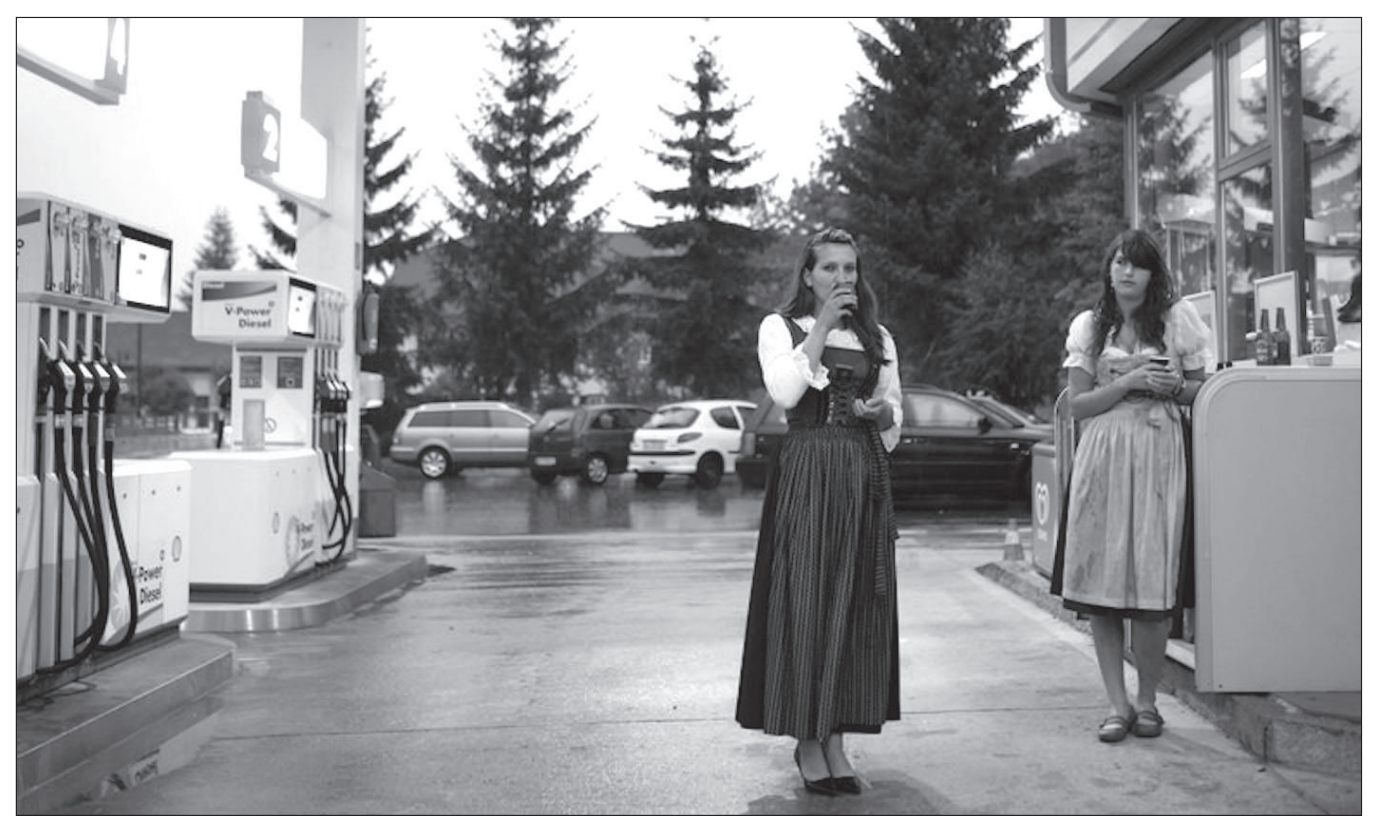

Abb. 1: "[Tiroler] Rennen nur in Trachten rum

Das stimmt nicht, obwohl es manchmal praktisch wäre. So ein Dirndl ist auch im Sommer luftig leicht (außer die traditionellen Trachten, welche meist aus schweren Stoffen genäht sind) und steht jeder Frau, ob rund, dünn, alt oder jung. Und die waschresistenten Lederhosen sind sowieso der Hit. Auf Zeltfesten und Prozessionen aber ist das Tiroler Gewand für jeden traditionsbewussten Einheimischen ein Muss. Vielerorts im Gastgewerbe unter den Kellnerinnen und Kellnern auch. Hier nicht immer ganz freiwillig. «

Zugleich wird hier behauptet, das »Tiroler Gewand « sei »für jeden traditionsbewussten Einheimischen ein Muss « - im Gastgewerbe unter den Kellnerinnen und Kellnern jedoch »nicht immer ganz freiwillig «. Interessant ist, dass selbst an dieser prominenten Stelle offiziösen PR-Handelns das Spannungsverhältnis zwischen Selbstbild und Fremdzuschreibung thematisiert wird. Die Tirol-Werbung befindet sich offenbar in einer Zwickmühle: Auf der einen Seite soll die Destination weiterhin als

I 3 https://www.blog.tirol/20 I6/o6/botschaft-uniform-heimat-historie-die-tracht/ (Zugriff: 21.04.2017) vgl. hierzu auch Egger, 2008.

I 4 https://www.blog.tirol/20I 5/o7/stimmt-oder-stimmt-nicht-wie-die-tiroler-soticken/ (Zugriff: 21.04.20I7) 
Hort naturnah-alpiner Urtümlichkeit positioniert werden (»Kraft- und Sehnsuchtsort im Herzen der Alpen $\ll),{ }^{15}$ gleichzeitig jedoch auch als Ort eines modernen, zeitgemäßen Lebensstils. Die mantraartige Wiederholung des angeblichen Gleich- und Einklangs zwischen der empirisch beobachtbaren gegenwärtigen Trachtenpraxis auf der einen und der touristisch und medial reproduzierten Klischeevorstellung auf der anderen Seite findet ihr semantisches Widerlager in der Modernitätsanrufung, die im Bild von den beiden Dirndlträgerinnen unter der Überdachung einer standardisierten Tankstelle eines global agierenden Ölmultis zum Ausdruck kommt. Dieser Spagat hinsichtlich der Selbstdarstellung zwischen Traditionalität und Modernität ist bekanntlich auch andernorts zu bewältigen und wurde etwa von den bayerischen Nachbarn seit Ende der I990er-Jahre mit dem Slogan » Laptop und Lederhose " adressiert. ${ }^{16}$

Auf der Ebene beobachtbarer Praktiken jenseits touristischer Vermarktung jedoch wird in Tirol eine Menge dafür getan, nicht die Dissonanzen dieser konfligierenden Bedeutungszuschreibungen in den Vordergrund zu rücken, sondern vielmehr Bilder von Stimmigkeit und Geschlossenheit zu transportieren. Dies betrifft insbesondere die Bereiche der so genannten Volkskultur, gemeint sind damit etwa Blasmusikkapellen, Volkstanzgruppen, die Tiroler Schützen ${ }^{17}$ und natürlich das organisierte Trachtenwesen, wobei sich auch die anderen Genres bei entsprechenden Anlässen in Tracht präsentieren. Der Eindruck der Geschlossenheit und der Uniformität in Habitus und Auftreten wird durch organisierte Vorführungen bei offiziellen Anlässen, durch choreografierte Aufmarschszenarien und auch durch möglichst einheitliche Kleidung im Trachtenstil erreicht.

I 5 http://www.tirolwerbung.at/marke-tirol/ (Zugriff: 2 I.04.20I7).

I 6 Vgl. Gabriele Wolf: Laptop und Lederhose, publiziert am 07.09.20 I2; in: Historisches Lexikon Bayerns, http://www.historisches-lexikon-bayerns.de/Lexikon/Laptop und Lederhose (Zugriff: 21.04.20I7).

I7 Dies sind in sogenannten Kompanien organisierte, mit repräsentativen Aufgaben bedachte Traditionsvereine, die anlassbezogen in choreografierten Formationen aufmarschieren, Salutschüsse mit Platzpatronen abgeben und die Wehrhaftigkeit und den Stolz des Landes symbolisieren - nicht zu verwechseln mit Sportschützenvereinen. Paradigmatisch für das Selbstverständnis ist die folgende Erklärung des Kommandanten anlässlich seiner Wiederwahl am 30.4.20I7: "Im Bewusstsein der historischen Verpflichtung um das Land sehen wir die Landesverteidigung heute im Eintreten für unsere Tiroler Identität - als Bewahrer und Hüter unserer Kultur und einer größtmöglichen Landeseinheit. Die Tiroler Schützen standen und stehen dafür ein, die Geschichte zu kennen, an der Zukunft zu bauen, die Tradition zu bewahren und Heimat zu geben!« Vgl. http://www.tt.com/panorama/ gesellschaft/I 2926502-9I/968-prozent-salut-für-tiefenthaler.csp (Zugriff: $05.05 .2017)$. 
Hinzu kommt noch die hierarchisch-korporative Organisationsstruktur mit unterschiedlichen Vereins- und Verbandsebenen und schließlich die nahezu unverhohlene Anbindung an die politische Sphäre, die ihren symbolischen Ausdruck insbesondere darin findet, dass der aktuelle Landeshauptmann von Tirol zwar nicht in seiner politischen Funktion, aber doch als Person Präsident des Tiroler Landestrachtenverbandes, des Tiroler Blasmusikverbandes und des Tiroler Sängerbundes ist. Diese gesamte Organisationsform zielt sowohl im Inneren auf eine hierarchische Strukturierung als auch nach außen, durch entsprechendes Gebaren und anlassbezogenes Auftreten, auf eine deutliche Nähe zur offiziös-politischen Sphäre der Macht. All dies findet seine habituellen Entsprechungen in quasi-militärisch anmutenden Aufmärschen und Traditionalität und Einheitlichkeit signalisierenden trachtigen Kleidungscodizes. Mit diesem organisatorisch-habituellen Komplex wird die staatstragende Bedeutung des Trachtenwesens symbolisiert und auch die nobilitierende Integration in das »Gesamtsystem Tirol « erreicht.

Seinen handfesten ökonomischen Ausdruck findet dieser Verflechtungszusammenhang in der finanziellen Förderung entsprechender Aktivitäten aus Steuermitteln, welche sich im Trachtenwesen unter anderem auf Veranstaltungsförderungen, Anschaffungs- und Erhaltungskosten von Trachten, Zubehör, Musikinstrumenten und Fahnen erstreckt. ${ }^{18}$ In einer eigens im Dezember 20I 5 erlassenen »Richtlinie zur Förderung der Kultur - Volkskultur « sind die diesbezüglichen Bestimmungen detailliert ausgeführt, interessant ist hierbei insbesondere der Befund, dass beide oben ausgeführten Verankerungsbehauptungen, die zeitliche wie die räumliche, in der Verwaltungsrichtlinie festgeschrieben sind: Unter $\mathbb{S} 2$ »Zielsetzungen " wird unter anderem formuliert, dass sowohl die regionale Verankerung (Abs. a) als auch die Pflege von Praktiken »mit historischer Kontinuität« (Abs. b) Kriterien für die Förderwürdigkeit von Einzelanträgen darstellen. ${ }^{19}$

Sowohl bei kirchlichen Anlässen (zum Beispiel Fronleichnamsprozessionen) als auch bei offiziellen Einweihungen oder staatlichen Feieranlässen (zum Beispiel Nationalfeiertag) wirken trachtentragende Schützen- und Blasmusikgruppen mit und geben dort nicht nur eine pittoreske Kulisse für das Geschehen ab, sondern erzeugen auch auf ästhetischer Ebene die Anmutung von Einheitlichkeit, Geschlossenheit und Kollektivität. Die ostentativ ausgestellte wechselseitige Partizipation von katholischer Kirche, öffentlicher Verwaltung, politischem Raum und Trachtenwesen resultiert in reziprokem symbolischen Profit und ge-

I 8 https://www.tirol.gv.at/kunst-kultur/kulturfoerderungen/heimat-undbrauchtumspflege/ (Zugriff: 21.04.20I7).

I9 https://www.tirol.gv.at/fileadmin/themen/kunst-kultur/abteilung/Sonstiges/ Richtlinie_Volkskultur.pdf (Zugriff: 21.04.20I7). 
genseitiger Relevanzversicherung. Der auf phänomenologischer Ebene dominierende Eindruck von Uniformität, Abgestimmtheit und geschlossenem Auftreten kann im Zusammenspiel mit der offiziösen Situierung der Auftritte in der Wirkung leicht in eine soziokulturelle Homogenitätsbehauptung umschlagen: auf Gruppenebene, auf Vereinsebene, auf Bevölkerungs- oder sogar Landesebene.

Ähnlich einer Uniform wirken Trachten nach innen homogenisierend und einheitsstiftend und kommunizieren diese Homogenitätsbehauptung zugleich dem Publikum. Dass sich das Bestreben, ein bestimmtes einheitliches Bild abzugeben, nicht nur auf die Kleidungsstücke selbst, sondern auf die Gesamterscheinung bezieht, ist auch daran erkennbar, dass andere Aspekte des Erscheinungsbildes wie etwa die Frisuren oder die Verwendung von Make-up oder Schmuck ebenfalls Gegenstand von Verhaltenskodizes und Reglementierungsbemühungen sind. In einem Artikel »Verwurzelte Tiroler und das Brauchtum « in der Erstausgabe des Tiroler Trachten-Zentralorgans "Der Trachtler ${ }^{20}$ aus dem Jahr I973 wird eine einheitliche Erscheinung, »ein ordentlicher Haarschnitt bei Männern und Frauen ", der Verzicht auf Armbanduhren und zurückhaltendes Make-up eingefordert: »Das auffällige Schminken des Gesichts und das Lackieren der Nägel paßt nicht zur Tracht und hat zu unterbleiben. " (Glatzl, I973, S. I2, vgl. auch Lentz in diesem Band, Abschnitt 2.2.). Und auch in dem aktuellen »Lernbehelf « für die Ablegung des »Volkskulturellen Leistungsabzeichens Bronze « finden sich Ausführungen, die in ähnlicher Weise Ordnung und Einheitlichkeit im Erscheinungsbild zur Maxime erklären und anmahnen: »Die Tracht als Ganzes hat in sich ein ausgewogenes Erscheinungsbild, das durch eine unpassende Frisur nicht zerstört werden soll (z.B. sollten lange Haare aufgesteckt oder geflochten werden). Aufdringliche und übertriebene modische Einflüsse stören die Harmonie « (Tiroler Landestrachtenverband, 20I4, S. 29).

Auch wenn zu vermuten ist, dass derartige Bemühungen um Einheitlichkeit und Disziplin auf der Regelungsebene immer auch auf gelegentliche Uneinheitlichkeit und Devianz auf der Praxisebene schließen lassen, so ist doch deutlich, dass insgesamt ein Eindruck der Geschlossenheit erweckt werden soll und in der Summe bei öffentlichen Auftritten auch erweckt wird. Gerade in diesen Homogenisierungsmomenten liegt der ästhetisch-semiotische Effekt des gesamten Trachtenwesens, das sich durch eine stimmig und konsequent durchchoreografierte Schauseite auszeichnet. Jenseits dieser auf Geschlossenheit und Einheitlichkeit zielenden Aktivitäten auf der Vorderbühne entspinnt sich jedoch ein kom-

20 Offizielles, seit I973 vierteljährlich mit einer derzeitigen Auflage von I700 erscheinendes Publikationsorgan des Tiroler Landestrachtenverbandes http:// www.landestrachtenverband.at/trachtler (Zugriff: 21.04.20I7). 
plexes und differenziertes, auf den ersten Blick gar nicht so einfach zu erkennendes internes Spiel aus Abgrenzungen und Zuordnungen auf den Hinterbühnen, das alle monochromen Vorstellungen einer einheitlich traditionsbewussten und monolithisch agierenden trachtig gewandeten Bergbevölkerung irritiert.

Während die Tracht sowohl in vielen medialen Repräsentationen als auch in der Homo Zillertaliensis-Miniatur aus dem Jahr 1974 treffsicher die Grenzlinie zwischen hiesig und fremd markiert, wenn auch unter den Verstärkungsbedingungen der Tourismusindustrie, so stellt sich das Geschehen gegenwärtig weitaus komplizierter und unübersichtlicher dar. Die Differenzierungskriterien verlaufen tatsächlich - wenn man so will - quer durch die Kleiderschränke und Jankersortimente der einschlägigen Ausstatter; das Trachtenwesen zeigt sich bei näherer Betrachtung als fragmentiertes Feld und differenzierter Mechanismus der Herstellung von Unterscheidungen. ${ }^{21}$ Im Folgenden werden drei derartige Verfahren des doing difference ausgeführt. In der Summe zeigen sich Tiroler Trachtenpraktiken als ebenso wirkmächtige wie ambivalente kulturelle Manöver der Herstellung und auch Irritation von Ordnungen, die sich weder auf einen Nenner bringen noch über einen Kamm scheren lassen, es sei denn es ist dieser: Trachten können vieles bedeuteten, aber nie nichts. ${ }^{22}$

Eines ist zudem klar: Das Tragen von Trachten ist eine Praxis mit ostentativem Charakter, das Moment des absichtsvollen und nachdrücklichen Zeigens ist ebenso offensichtlich wie inhärent. Zugleich ist die hiermit verbundene Botschaft durch den Aspekt der Kleidung, der sich ja unmittelbar auf die sie tragende Person bezieht, unausweichlich reflexiv-identitär gewendet. Es ist klar, dass mit dem Tragen von Tracht nicht nur etwas ausgesagt werden soll, also eine Mitteilung, sondern insbesondere auch etwas über die Tracht tragende Person. Dies ist zwar auch bei anderen semiotisch befrachteten alltagskulturellen Praktiken der Fall, doch in Kleidungsangelegenheiten ist dieser Effekt der reflexiven Stilisierung mit personaler Akzentuierung besonders ausgeprägt. Turnschuhe bei der Vereidigung als Minister, zerrissene Jeans vor Gericht oder der beste Anzug bei der Wohnungsbesichtigung beziehungsweise beim Vorstellungsgespräch - dies alles sind starke Zeichen, die sich zwar auch auf die Situation beziehen, dabei jedoch gezielt die jeweilige Person als solche mit einbinden. Im Fall der Tracht ist dieser

2I Einen unterhaltsamen und bildgewaltigen Einblick in die Mehrdeutigkeiten und Lesarten dieser Kleidungspraktiken bietet mit Blick auf viele Gebiete Österreichs: Stoff der Heimat. Ein Dokumentarfilm von Othmar Schmiderer (Regie) und Elsbeth Wallnöfer (Konzept), Österreich 20I I; vgl. http://www. stoffderheimat.at/ (Zugriff: 21.04.20I7).

22 Vgl. Marsel, 2016, insbes. S. I68: »Kleidung schweigt nicht.« 
Mechanismus zusätzlich gesteigert, weil häufig mit einem Verweis auf Aspekte der persönlichen Haltung, der Einstellung oder gar des Charakters verbunden. Nicht umsonst ist bei Trachten auch immer wieder von einem »Bekenntniskleid ${ }^{23}$ die Rede. Insofern ist es nur folgerichtig, die Praxis des Trachttragens unter dem Aspekt der Humandifferenzierung zu begreifen beziehungsweise als solche - und damit als spezifische Form der Herstellung von sozialer Ungleichheit - zu analysieren (vgl. Hirschauer in diesem Band, Abschnitt 3). Weil aber alles eine Frage der Kontexte ist und jene vielfältig sind, gilt es nun, im Feld der Trachtenpraxis verschiedene Verfahren der Zugehörigkeitsherstellung zu unterscheiden und jeweils an empirischem Material zumindest exemplarisch nachzuzeichnen. Auch wenn derartige Verfahren der Herstellung von Zugehörigkeiten immer mit einer Doppelbewegung aus Abgrenzung und Zuordnung verbunden sind, erfolgt die Darstellung hier unter drei Begriffspaaren, die jeweils einen Aspekt des doing difference markieren: Trachtler und Nicht-Trachtler, Trachtler und Trachtige, Qualitätsbewusste und Fabriksware«-Tragende.

\section{Trachtler und Nicht-Trachtler}

Die offensichtlichste und augenscheinlichste Markierung ist kurioserweise zugleich die am schwierigsten zu charakterisierende, nämlich die zwischen denjenigen Personen, die eine Tracht im Kleiderschrank haben und diese auch - regelmäßig, gelegentlich oder selten - bei öffentlichen Anlässen tragen, und denjenigen, für die dies nicht gilt. Es gibt also Trachtenträger/innen, in der Selbstbezeichnung auch »Trachtler ", und einen nicht trachtenaffinen » Rest « der Welt. In dieser allgemeinen Form erscheint diese Unterscheidung simpel und klar, sie entspricht auch einer weit verbreiteten und - wie oben gezeigt - von der Tourismuswerbung immer wieder sorgsam gehegten und gepflegten Vorstellung vom vestimentären Treiben in alpinen Regionen: Da gebe es eben welche, die » in Tracht rumlaufen «.

Doch allein schon die Frage nach der Quantifizierung dieser Gruppe wirft Probleme auf und führt damit zugleich mitten in den Dschungel der Binnendifferenzierungen und Definitionsprobleme. Wie viele »aktive Trachtler « gibt es eigentlich in Tirol - genauer: dem österreichischen

23 https://schuetzen.com/druckansicht/aktuellbericht/artikel/vortrag-die-trachtist-in-342.html (Zugriff: 21.04.20I7). Wobei hier der Vollständigkeit halber betont werden muss, dass der Begriff »Bekenntniskleid « oft und auch hier in einem Südtiroler Kontext mit der spezifischen Bedeutung des Bekennens zum Deutschsein vor dem historischen Hintergrund der repressiven Italianisierung verbunden war und ist. 
Bundesland Tirol mit seinen rund 740.000 Einwohnern, das auch den Bezirk Osttirol, nicht jedoch das politisch Italien zugehörige Südtirol umfasst? Diese Frage ist schlichtweg nicht zu beantworten; sogar jene nach der Anzahl der Tiroler Brillenträger/innen oder Nichtschwimmer/ innen wäre vermutlich einfacher anzunähern, denn die Schwierigkeit beginnt schon dabei, dass es weiterhin definitorisch ungeklärt und auch strittig ist, was eigentlich eine Tracht sei. Während manche schon eine singuläre Lederhose oder eine Strickjacke im Landhausstil mit Zopfmuster und Alpenblumen-Applikation hinzurechnen, fallen derartige Kleidungsstücke für andere noch lange nicht unter den Trachtenbegriff. Ganz zu schweigen von der Frage, in wie vielen Tiroler Kleiderschränken sich derartige Stücke finden. Worüber es allerdings relativ verlässliche Zahlen gibt, ist die Anzahl an Mitgliedern in jenen rund I05 Vereinen, die im Tiroler Landestrachtenverband zusammengeschlossen sind: ca. I0.000, also rund I,4 \% der Bevölkerung.. ${ }^{24}$ Aber was heißt das schon? Quantitativ ist dies auf die Gesamtbevölkerung gesehen eine kleine Minderheit und auch für die Frage der Verbreitung der Trachtenidee und Trachtenpraxis allenfalls ein erster Anhaltspunkt.

Aufschlussreich ist jedoch, welche inhaltlichen Zuschreibungen der in der Selbstcharakterisierung »oberste « und »durch und durch überzeugte " Trachtler Tirols, ${ }^{25}$ der Landesverbandsobmann in Bezug auf das Trachtenwesen vornimmt: Es gehe um »Heimatverbundenheit, Bodenständigkeit und Geborgenheit « (Darnhofer \& Rapp, 2015, S. 3), um einen Mix aus Haltung, Emotion und Charakter also, der mit dem »Kleid der Väter « (Darnhofer \& Rapp, 20 I 5, S. 5) nicht nur zum Ausdruck gebracht, sondern auch ins Werk gesetzt werde. Bei genauerer Betrachtung jedoch bleiben auch diese inhaltlichen Zuschreibungen eigentümlich unklar, sie skizzieren eher einen Bedeutungs- und Assoziationsraum, auf den das Zeichensystem Tracht angeblich referiert. Wie und warum dies genau so sei, bleibt jedoch im Vagen. Das, was »die Trachtler « jenseits der Tatsache, dass sie Tracht tragen, eigentlich im Kern zusammenhält oder eint, bleibt unbestimmt und erscheint letztlich als eine Art Arkanwissen der an dieser Praxis Teilhabenden - wer es nicht selbst tut, wird es nie ganz verstehen können. In programmatischen Formulierungen wie etwa der, dass es um die »Wahrung und Förderung des heimischen Trachtenwesens und des Brauchtums im Jahreskreis ${ }^{26}$ gehe, wird neben einer Drift zur Tautologie (»Trachtler trachtlern«) allenfalls ein kuratives Moment der Wahrung und der Pflege deutlich, das sich »in Zeiten

24 Darnhofer/Rapp 20I 5, 2; auch http://www.landestrachtenverband.at/ (Zugriff: 21.04.2017).

25 http://www.landestrachtenverband.at/vorstand/Io-landesverbandsobmann (Zugriff: 2I.04.20I 7 ).

26 http://www.landestrachtenverband.at/ (Zugriff: 21.04.20I7). 
wie diesen « (Darnhofer \& Rapp, 20 I 5, S. 3 ) den Dynamiken moderner Beliebigkeit und den im fliegenden Galopp davoneilenden Signifikanten irgendwie entgegenstelle. In solchen Verlautbarungen konturiert sich die Trachtenszene - zumindest die organisierte - gerne als ein Fels in der post- oder spätmodernen Brandung und somit als ein soziales und vereinsmäßiges Angebot, das von »der Jugend « gerne angenommen werde.

Das auf diese Weise evozierte Bild einer dichotomen Gegenüberstellung von einem System personaler, sozialer und gesellschaftlicher Verlässlichkeit und Solidität auf der einen Seite - repräsentiert durch die Trachtler - und den Dynamiken und Zentrifugaleffekten zeitgenössischer Diversifizierung und Destabilisierung auf der anderen Seite relativiert sich jedoch recht schnell, wenn man sich, wie unten ausgeführt werden wird, die Heterogenität dessen, was alles unter dem Begriff »Tracht « fungiert, vor Augen führt und einen Blick auf die vielfältigen und ebenfalls dynamischen internen Fragmentierungen des nur scheinbar monolithischen Trachtenblocks wirft.

Das organisierte Trachtenwesen selbst befindet sich argumentativ in einer gewissen double-bind-Situation. Auf der einen Seite wird die Trachtenpraxis als performative Vergegenwärtigung des Traditionalen und Hiesigen, also des raumzeitlich unzweifelhaft Zugehörigen und Verorteten konzipiert. Gerade Formulierungen wie die oben zitierten im Umfeld der Wurzelmetaphorik bringen diese Tendenz zur Naturalisierung und Essentialisierung deutlich zum Ausdruck. Auf der anderen Seite jedoch wird immer wieder betont, welche wichtige sozialintegrative Rolle die Trachtenvereine spielen können und welche konstruktiven Wirkungen diese gerade in Prozessen gesellschaftlicher Dynamisierung und Pluralisierung entfalten.

Auf dem Höhepunkt der oben erwähnten medial ausgetragenen und politischen Auseinandersetzung um nationalistische Implikationen und nationalsozialistische Vorgeschichten des Tiroler Trachtenwesens bezeichnete der Obmann des Landestrachtenverbandes im Jahr 2013 in einem Zeitungsartikel, die » Trachtler von heute " als »weltoffene, soziale und tolerante Menschen " (Abb. 2).

Wie als eine Art Beleg für diese These und auch die sozialintegrative Funktion der Vereine wurde der Artikel dann mit einem Foto bebildert, das vier Kinder in Tracht zeigt, wovon eines dunkelhäutig ist. Gerade vor dem Hintergrund der regionalen Debatte um historisch angelegte, latent weiterhin vorhandene und wirksame Exklusionseffekte im Feld der Volkskultur standen die Traditionsverbände unter Rechtfertigungs- und Erklärungsdruck. Die konkrete Anschauung und lokale Praxis, wie sie bei Festen, Umzügen und öffentlichen Anlässen in Tirol erlebbar sind, stützt durchaus den Eindruck, dass diese Praxisfelder soziokulturell von der angestammten Landbevölkerung dominiert werden und - wenn auch nicht programmatisch, so doch im realen Vollzug - von einer Maxime des Un- 

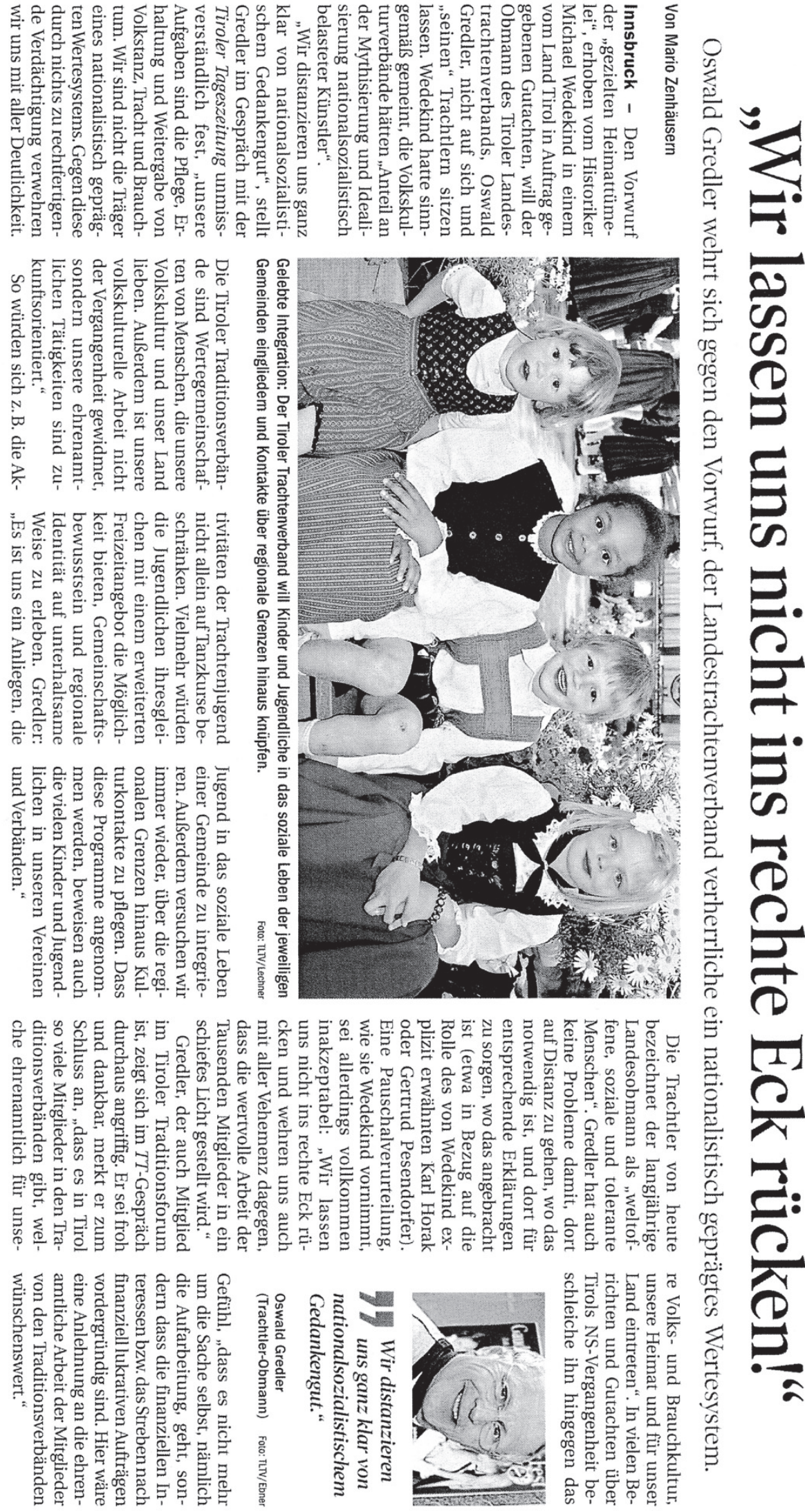

Abb. 2: "Wir lassen uns nicht ins rechte Eck rücken! « Artikel in der Tiroler Tageszeitung Nr. 283 vom 12.10.2013, S. 6. 
ter-sich-bleibens gekennzeichnet sind. Das Bild, das nicht nur in der Zeitung sondern auch auf dem Cover der Zeitschrift »Trachtler " eingesetzt wurde, fungiert in diesem diskursiven Kontext als eine Art Gegenbeweis gegen den Vorwurf einer Ausschlusspraxis entlang ethnischer Kategorien.

Kultursemiotisch betrachtet ist diese Text-Bild-Kombination insbesondere deshalb aufschlussreich, weil die offenbar intendierte Botschaft, es komme im Trachtenfeld letztlich gar nicht auf Hautfarbe und Herkunft an, gerade in ihrer ostentativen Platziertheit unweigerlich auf ihr Gegenteil verweist. Das lächelnde dunkelhäutige Mädchen inmitten seiner Vereins- und Altersgenossinnen erscheint genau deshalb als Sonderfall, weil es als Illustrierung mit Belegcharakter für eine apologetische Gegendarstellung auf Textebene fungiert. Der hier praktizierte demonstrative Entkoppelungsgestus von Trachtenpraxis und ethnischer Selbstverortung verweist auf den Regelfall der Situierung des Trachttragens im Milieu ortsansässiger Hellhäutiger (so wie auch in anderen Gegenden der Welt Tracht oft stark ethnisch codiert ist, sogar wenn dies eher unerwünscht ist, vgl. Lentz in diesem Band Abschnitte 2.2 und 3).

Noch deutlicher, weil zugleich performativ eingebettet und situativ erlebbar, erscheint dieser Effekt im Kontext einer Aufnahme, die in einer feldforschenden Beobachtungssituation bei einem Umzug auf einem Handwerksfest in Seefeld, einer Tiroler Gemeinde in der Nähe von Innsbruck entstand. Auf dieser dokumentarischen Fotografie ist zu sehen, wie die Trachtengruppe "D'Koasara Kufstein « von einem dunkelhäutigen Buben als Schildträger angeführt wird:

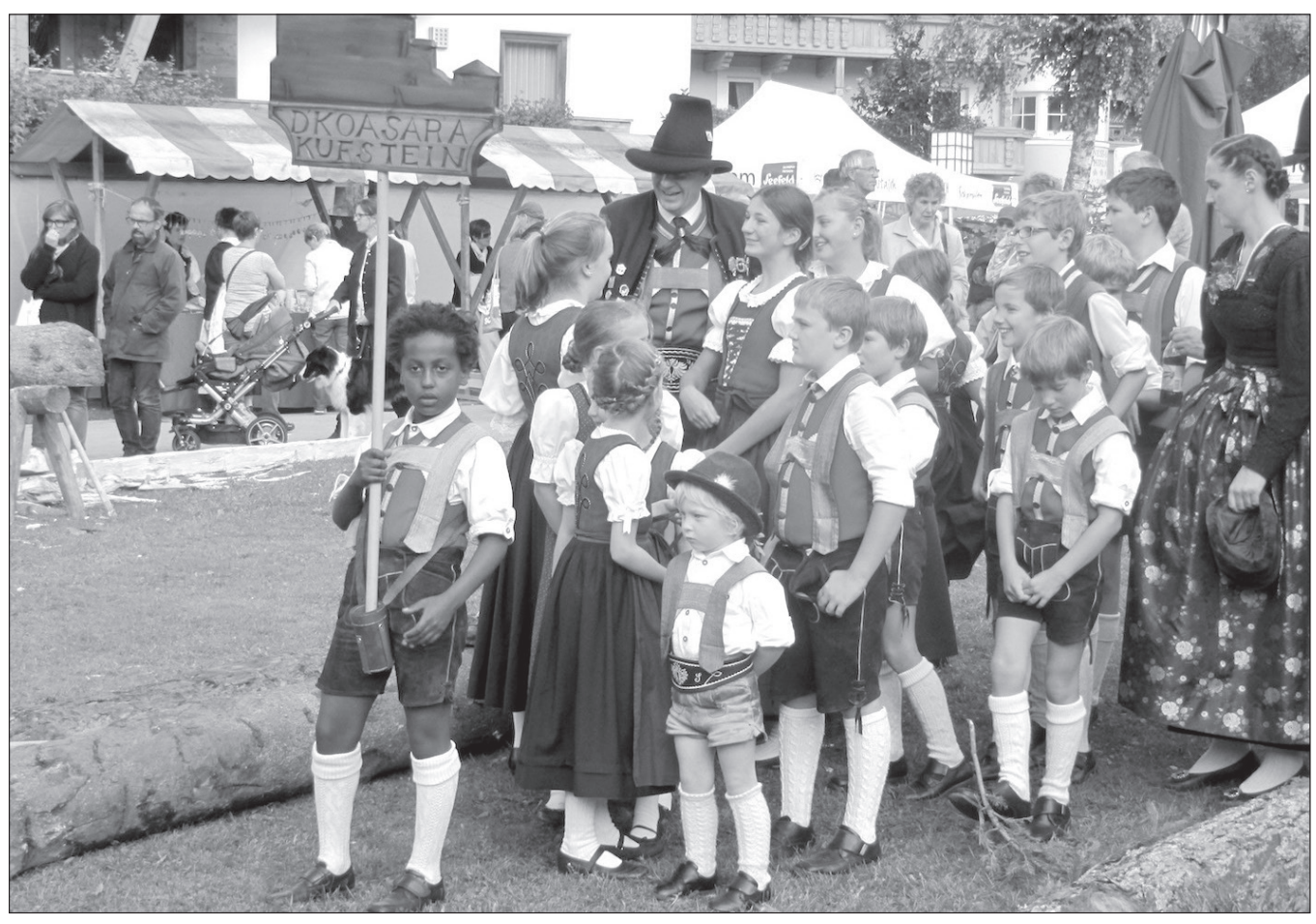

Abb. 3: Handwerksfest Seefeld, 14. September 2014 (Foto: Reinhard Bodner) 
Die Position und Rolle des Schildträgers ist bei derartigen Anlässen eine besonders exponierte und daher ehrenvolle; die Betrauung des Jungen mit dieser Aufgabe ist daher als ein starkes und bewusst gesetztes Zeichen des Vereins zu lesen. Der Junge ist in der Gruppe der einzige Mensch mit dunkler Hautfarbe, ein Eindruck der sich auch bei der Durchsicht des Bildmaterials auf der Homepage des Vereins bestätigt. ${ }^{27}$ Insofern ist die Positionierung des Jungen bei diesem öffentlichen Anlass an hervorgehobener Stelle tatsächlich ein starkes und aussagekräftiges Statement, das durchaus als Botschaft der Wertschätzung und demonstrative Beteuerung des Vereins zu lesen ist, keine nach ethnischen Kriterien geschlossene Gruppe sein zu wollen. Die dunkle Hautfarbe ist ganz offensichtlich für die Zugehörigkeit und auch die Möglichkeit, den Verein öffentlich zu repräsentieren, kein Hinderungsgrund, sie sei für diese Frage nicht relevant - diese Botschaft könnte man zumindest verstehen. Durch die Exponiertheit des - offenbar einzigen - dunkelhäutigen Jungen an der Spitze des Zuges wird diese Irrelevanzbehauptung performativ jedoch konterkariert bis aufgehoben, denn dieses Arrangement unterstreicht geradezu die Differenz der Hautfarbe und macht diese dadurch nolens volens zu einem hervorgehobenen Kriterium. Diese gesellschafts- und vereinspolitisch intendierte Geste des vorgeblichen undoing difference erweist sich also gerade als Dementi paradoxerweise tatsächlich in Praxis und Wirkung als ein Manöver der starken Hervorhebung von Differenz. Die Regularität der in aller Regel und üblicherweise erfolgenden ethnischen Situierung der Tiroler Trachtenpraxis im Kontext hellhäutiger Personengruppen wird dadurch ganz besonders unterstrichen, der eine dunkelhäutige Junge lässt die restlichen Trachtler erst recht hellhäutig erscheinen.

Natürlich befinden sich die Vereinsverantwortlichen in einer geradezu unlösbaren Zwickmühle, denn solange es in der gesamten Gruppe nur eine dunkelhäutige Person gibt, bleibt dieser Umstand immer als Differenzverstärker interpretierbar, ganz egal wo der Junge platziert worden wäre: An der Spitze der Gruppe fällt er unter den Verdacht, als antirassistisches Feigenblatt funktionalisiert zu werden, inmitten der Gruppe würde er »zurück ins Glied « geschickt (»Klar, dass er nicht an der Spitze gehen darf! «) und als massive Ausgrenzung würde es gelten, wenn er am Umzug gar nicht aktiv beteiligt würde. Man würde also dem kritischen Beobachter, in dessen Augen solche Effekte entstehen können, in jedem Fall Anlass zur Interpretation geben, die Sache bleibt ambivalent. Ein tatsächliches undoing difference im Sinne eines »Einschlafens " oder allgemeinen irrelevant-Werdens der Hautfarbe scheint in diesem konkreten Fall kaum möglich. Es würde Zeit und veränderte Sehgewohnheiten des Publikums verlangen.

27 http://www.koasara.at/ver2/index.php/jugendgruppe (Zugriff: 2I.04.20I7). 
Genau dies spiegelte sich auch in Kommentaren einzelner Besucherinnen, die bei der Veranstaltung aufzuschnappen waren: Während manche den Anblick goutierten und es als "ach, wie nett! « begrüßten, dass der kleine dunkelhäutige Junge das Schild tragen »durfte «, äußerten sich andere unverhohlen rassistisch: »Haben die Kufsteiner eigentlich nicht genügend Leute, so dass sie jetzt sogar schon einen Neger vorneweg laufen lassen müssen? « In beiden Fälle kommt zum Ausdruck, dass die Vorstellung sozialräumlicher Essentialisierung in Bezug auf Tiroler Trachten weiterhin in Kraft ist: Der Anblick eines dunkelhäutigen Jungen in alpiner Tracht war nicht nur ungewohnt, sondern stellte auch eine ebenso ungeschriebene wie offenbar gültige Regel der ethnischen Korrektheit in Frage, selbst wenn dieser Regelverstoß im Einzelfall sogar begrüßt worden sein mochte. Sowohl dieses positive Überraschungsmoment als auch sein rassistisch-abwertendes Pendant verweisen auf die offensichtlich stattgefundene Irritation einer in Funktion befindlichen Regel der Kopplung des Tiroler Trachtenkonzepts an ethnische Kategorien.

Es gibt demnach sowohl aus der Innen- als auch aus der Außenperspektive Trachtler und Nicht-Trachtler, und dies hat nicht nur mit der vestimentären Praxis zu tun, so wie man etwa durch das Tragen einer Krawatte zum Krawattenträger oder das eines Pelzmantels zur Pelzträgerin werden kann. Im Fall des Trachtler-Seins werden offenbar zudem noch ethnische und regionale Faktoren als relevant erachtet, es reicht nicht einfach aus, »in Tracht rumzulaufen ". Nicht jede beliebige Person kann einfach so Trachtler/in werden, denn manche Mitglieder eines sich als zuständig verstehenden Akteurs- und Zuschauerkollektivs beanspruchen in dieser Sache ein Mitsprache- oder gar Vetorecht.

\section{Trachtler und Trachtige}

Während die Scheidung in Trachtler und Nicht-Trachtler allgemeiner Natur ist und sowohl aus der Innen- als auch der Außenperspektive erfolgt, ist die Binnenwahrnehmung innerhalb der Trachtenszene noch wesentlich differenzierter. Sie findet entlang der Unterscheidung zwischen »echten Trachtlern « und lediglich »trachtig « Gekleideten ihren wichtigsten Ausdruck. Im Zentrum dieser Unterscheidung steht die Aushandlung dessen, was »ernsthafte " und »richtige « Trachtenpflege, und was nur »Trachtenmode " oder »Oktoberfestdirndl-Aufzug « sei. Maßgeblich stehen sich hier Ernsthaftigkeit und »Spaß « gegenüber; es wird um interne Organisation und die Hierarchisierung der zugeschriebenen Nähe zu einem wie auch immer gearteten Authentizitäts- oder Ernsthaftigkeitskern gerungen.

Die Frage danach, welche Tracht für eine bestimmte Person, eine bestimmte Region oder einen bestimmten Anlass die »richtige «, »korrek- 
te « oder " authentische « sei, ist so alt wie der »Denkstil Tracht « selbst. Sie zieht sich wie ein roter Faden durch die Trachtengeschichte der letzten rund 200 Jahre, zumindest jedoch durch jene Phase seit Mitte des I9. Jahrhunderts, die mit der Erfindung und Entdeckung des Konzeptes Volkskultur, des Faches Volkskunde und allen angelagerten Musealisierungs-, Sammlungs-, Forschungs-, Vereins- und Verwaltungsaktivitäten verbunden ist. Im Zentrum dieser Frage nach der »richtigen Tracht « steht zugleich das Konzept der » Trachtenberatung «, für welche in der Vergangenheit sowohl in Österreich wie auch in Deutschland, vornehmlich in Bayern, teilweise eigene Stellen geschaffen wurden oder die als konkrete Anleitungs- und Normierungspraxis von Institutionen und Personen entweder bereitwillig übernommen oder auch verweigert wurde. Es sind hiermit Serviceleistungen gemeint, die Ratsuchende - Vereine oder Einzelpersonen - hinsichtlich Schnittmuster, Materialwahl und Anfertigung instruieren und somit nicht nur praxiswirksam anleiten, sondern auch normierend wirken und insgesamt einem statisch-konservierenden Kulturverständnis Vorschub leisten.

In Tirol war für derartige Zertifizierungsdienste seit den I920er Jahren das Tiroler Volkskunstmuseum die erste Adresse (Abb. 4), diese Aktivität ist eng mit den Namen Josef Ringler und Gertrud Pesendorfer verknüpft, wurde aber auch noch bis in die s98oer Jahre fortgeführt, wenn auch zunehmend widerwillig. Denn in dem Maß, in dem sich in der wissenschaftlichen Volkskunde immer mehr die Auffassung durchsetzte, dass eine derartige Beratungspraxis mit den Einsichten und Zielen einer konstruktivistisch orientierten modernen Kulturwissenschaft eigentlich unvereinbar war, zog sich das Museum aus dieser Tätigkeit zunehmend zurück. Dies wiederum gab privaten oder vereinsmäßig organisierten Initiativen der Beratungspraxis Raum ${ }^{28}$ und beförderte zudem innerhalb des Verbandwesens Bestrebungen der Institutionalisierung und Normierung: Seit 1987 wird vom Landestrachtenverband das bereits erwähnte sogenannte »volkskulturelle Leistungsabzeichen « in den drei Schwierigkeitsklassen Bronze, Silber und Gold als Ausbildungsprogramm angeboten beziehungsweise verliehen (vgl. Bodner, 20I7).

Dieses hierarchisierte Kurs- und Auszeichnungssystem, analog zum Leistungsabzeichen des Tiroler Blasmusikverbandes ${ }^{29}$ konzipiert, war

$28 \mathrm{Zu}$ nennen sind hier insbesondere die Arbeitsgemeinschaft (ARGE) »Lebendige Tracht und Volkskultur (innerhalb der Tiroler Heimatpflege, Gründung I979 vgl. http://www.heimatschutzverein.at/arbeitsgruppen_lebendige-tracht.php, die ARGE »Lebendige Tracht in Südtirol « (Gründung I980 vgl. http://www.hpv.bz.it/de/arbeitsgemeinschaft-lebendige-tracht.html Zugriff: 21.04.20I7) und als jüngste Gründung 2007 die »Arbeitsgruppe Unsere Tracht« (vgl. http://www.unsere-tracht.info/).

29 http://www.blasmusik.tirol/fachbereiche/jugendreferat-blasmusikverband-tirol/leistungsabzeichen.html. 


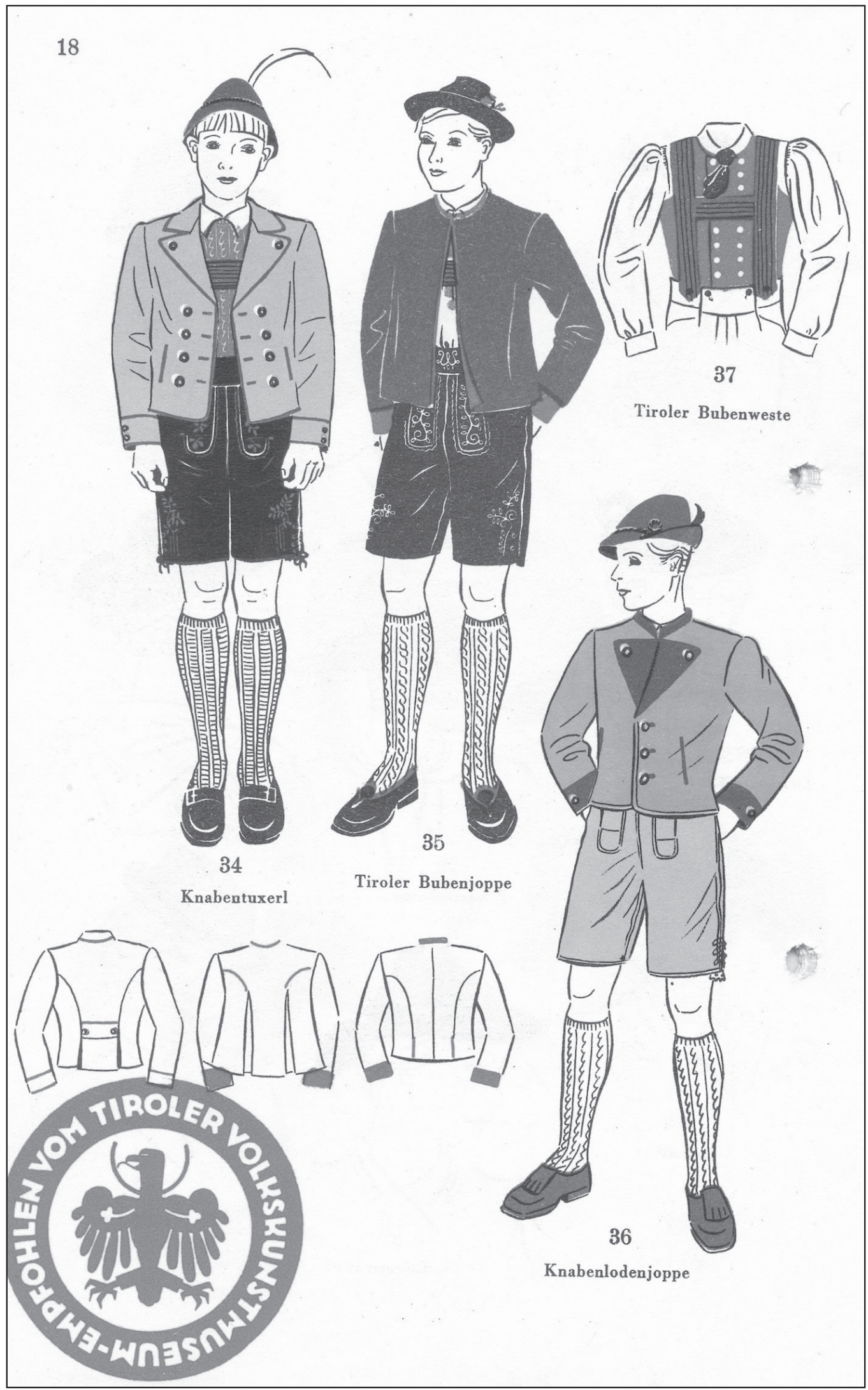

Abb. 4: »Empfohlen vom Tiroler Volkskunstmuseum « - Beispielseite aus dem Katalog »Lodenbaur Trachtenbekleidung", Innsbruck 1936 (Archiv des Tiroler Volkskunstmuseums) 
einerseits als Mittel zur Hebung und Sicherung der »Qualität « des in den Vereinen zirkulierenden Wissens über Trachten und Brauchtum gedacht, muss jedoch auch im Kontext des Bemühens der Trachtenvereine um Rang und Status im Vergleich zu anderen volkskulturellen Genres verstanden werden, denn ein historisch wichtiges, die vergangenen 30 Jahre betreffendes Moment ist das Ringen der Trachtler um soziale Anerkennung, Aufwertung und Gleichstellung im Feld volkskultureller Praxen. Viele Jahre lang galten die Trachtler - verglichen mit den Schützenkompanien, den Musikkapellen oder den Volkstanzgruppen - als das rangniedrigste volkskulturelle Genre. Die Trachtler beklagen, teilweise noch bis in die jüngste Vergangenheit als »die Schuhplattler « nur nachrangige Wertigkeit beanspruchen zu können. Auch wenn sie nicht offen verlacht wurden, so fühlten sie sich doch über weite Strecken als »die Dodeln « geringgeschätzt, die zwar mit unterhaltsamen Auftritten aber nur wenig symbolischem Kapital aufzuwarten hatten..$^{3 \circ}$ Das volkskulturelle Leistungsabzeichen sollte hier Abhilfe schaffen. Inhaltlich-strukturell handelt es sich bei diesem Ausbildungs- und Zertifizierungssystem aus kulturanalytischer Perspektive um ein Normierungs- und Standardisierungsprogramm der positivistischen Festschreibung kanonisierter Kulturphänomene. Dies wird auch in der Selbstdarstellung des Verbandes deutlich: »Bei dieser Prüfung wird das Wissen über Tracht, Brauchtum und Volkstanz überprüft - das Niveau des volkskulturellen Leistungsabzeichens ist sehr hoch. So gehören die Tanzerklärung versch. Tänze, genauso wie die Trachtenerkennung und -erklärung, sowie die Tiroler Bräuche ebenso zum Prüfungsumfang wie der praktische Teil mit der Vorführung einiger Tänze, die per Los aus dem großen Tanzspektrum gezogen werden. « $^{3}$

Differenzierungstheoretisch ist dieses Zertifizierungssystem sowohl in der Innen- als auch der Außenwirkung von großer Bedeutung. Nach außen weisen sich die Leistungsabzeichenträger/innen durch sichtbares Tragen bei öffentlichen Anlässen als Fachleute aus. Nach innen resultiert dieses System in einer Rangordnung der Kenner- und Könner-

$30 \mathrm{Vgl}$. folgende beide Zeitungsartikel: Trachtler haben das >Dodel-Klischee satt. «In: Tiroler Tageszeitung, 29.0I.I998, S. I 2 und Robert Benedikt: »Wir wollen nicht mehr die Dodeln sein«. In: Die Presse, 03.03.I998, Rubrik »Journal«, S. I 2 .

3 I http://www.landestrachtenverband.at/vorstand/landesjugendreferat/4-landesjugendreferat (Zugriff: 2I.04.20I7). Die Struktur und Genese der schriftlichen Lernbehelfe, das Zustandekommen der prüfungsrelevanten Inhalte, die institutionelle Rahmung der Prüfungen und die komplexe Verschränkung des gesamten Unterfangens mit der Geschichte der akademischen Volkskunde an der Universität Innsbruck ist ein eigenes Thema, das hier nicht ausgeführt werden kann, ich verweise dazu auf die noch ausstehenden Publikationen von Reinhard Bodner aus den laufenden Projektarbeiten. 
schaft, die prinzipiell für jeden offen ist, leistungsbasiert durchlaufen beziehungsweise erklommen werden kann und eine strikt pyramidale Struktur aufweist. ${ }^{22}$ Gegenwärtig (Stand April 20I7) gibt es 944 bronzene, 546 silberne und 65 goldene Leistungsabzeichenträger/innen. Vereinsintern ist auch allgemein bekannt, wer welches Abzeichen erworben hat. Insofern ist tatsächlich Trachtler nicht gleich Trachtler, sondern es besteht entlang den Zertifizierungskategorien ein hierarchisch gegliedertes Klassifikationssystem der Zuschreibung von Kompetenz, Fachwissen und Status. Die Abzeichen sind als Objektivationen sowohl konkreter Ausdruck eines Bildungs- und Wissensaneignungsprozesses als auch damit unmittelbare emblematische Verweise auf Kategorien wie Ernsthaftigkeit oder Kompetenz und begründen damit Ansprüche auf den szeneinternen Rang, der dann praktisch im Austausch von trachtenbezogenem Detailwissen und Fachsimpeleien seine performative Bestätigung erfährt.

Diese feldinterne Expert/innenposition ist jedoch, dies sei hier der Vollständigkeit halber betont, nicht nur auf dem Weg des Leistungsabzeichenerwerbs zu erlangen. Nadja Neuner-Schatz kann in ihrer Ethnografie eines Trachtennähkurses zeigen, wie ein ganzes Bündel aus Praktiken des Wissens, des Erzählens, der Beratens, der Urteilens und nicht zuletzt auch des praktischen Anfertigens ebenso dazu geeignet ist, Autorität in Trachtenfragen herzustellen und zu sichern, allerdings eben im speziellen Kontext eines Nähkurses (Neuner-Schatz, 2016, S. I34). Im Bereich des organisierten Verbandswesens spielt das Leistungsabzeichen in seiner ordensartigen Emblematik jedoch eine wichtige Rolle. Zugleich präsentieren sich die Vereine und der Dachverband samt seinen Funktionsträgern auf diese Weise nach außen - und das heißt hier: in der Öffentlichkeit - gewissermaßen als Hüterinnen von Tradition und »reiner Lehre " gegenüber einer zeitgenössischen Gesellschaft, die entweder ablehnend oder indifferent auf das Trachtenthema reagiert oder aber, weitaus heikler, in vielfältiger Art und Weise einzelne trachtige Elemente in ihrer Freizeit- und Feierkultur (»Oktoberfestdirndl «) verarbeitet und damit gleichsam spaßkulturell verwertet und verdaut (vgl. Egger, 2008).

Diese Aneignungsformen stellen als vordergründige Spaßkultur ohne Substanz für die um Traditionalität und Ernsthaftigkeit bemühte or-

32 Demonstriert wurde diese prinzipielle Offenheit im Jahr $20 \mathrm{I} 2$ mit dem medial-öffentlichen freudigen Verweis darauf, dass erstmals »ein Niederländer « die »Plattlermatura « bestanden habe. Analog zu dem oben ausgeführten Beispiel wird hier durch ein performatives Dementi eines nationalen Ausschlusskriteriums die weiter fortbestehende grundsätzliche Relevanz der Differenzkategorie »Nationalität« zugleich unterstrichen: https://www.meinbezirk. at/hall-rum/leute/erster-niederlaender-besteht-plattlermatura-d I $57 \mathrm{I} 63 . \mathrm{html}$ (Zugriff: 21.04.2017). 
ganisierte Trachtenszene ein gewisses epistemisches Problem dar: Einerseits bemüht sie sich, diesen Phänomenen ihr eigenes Konzept von Dignität und Seriosität der Tracht entgegenzuhalten, andererseits wird das immer wieder gescholtene »Dirndl« durchaus im positiven Sinne als »Einstieg " wertgeschätzt, das »jungen Leuten « einen Zugang zum Thema der Tracht eröffnen könne, der dann freilich unter den qualitätssichernden Fittichen der vereinsmäßigen Organisation gezielt weiterentwickelt und veredelt werden müsse (Darnhofer \& Rapp, 20I 5, S. 5).33

\section{Qualitätsbewusste und Fabriksware«-Tragende}

Mit dem Begriff der »Qualität « ist zugleich das Stichwort für die letzte hier noch kurz zu behandelnde Unterscheidung genannt, die in doppelter Hinsicht ins Innere zielt: in das der bereits abgegrenzten Gruppe der »ernsthaften Trachtler " und in das der Kleidungsstücke selbst, ihre Herstellung und Materialität. Es geht um die Frage der Stofflichkeit, der Stoffe und der Machart, mithin um die der Güte, Beschaffenheit und - noch einmal - der Authentizität, allerdings eben nicht in formaler Hinsicht, sondern in materiell-qualitativer.

Eng mit diesem Qualitätsaspekt und der Frage der Herstellung verknüpft ist die Ökonomie, denn Handarbeit und edle Materialien kosten mehr als industriell hergestellte Textilien und maschinelle Fertigung. Und hiermit ist auch eine soziale Dimension berührt, denn es stellt sich rasch die Frage, wer sich eigentlich welche Tracht leisten kann. ${ }^{34}$ Es geht also ums Eingenähte. Deshalb birgt diese Unterscheidungsfrage unter den hier besprochenen Differenzierungsmanövern das vielleicht größte Konfliktpotenzial mit sich, weil es hier nicht in erster Linie um die Abgrenzung gegen ein ungläubiges oder ignorantes Außen geht, sondern auch um Fragen der Inklusion und Exklusion innerhalb des Feldes der Trachtenaffinen. In ihrer schärfsten Zuspitzung werden diese Fragen in szeneinternen Auseinandersetzungen mit Begriffen wie Treue und Verrat verhandelt und kommen damit schon in die Nähe der Kategorien von Rechtgläubigkeit oder Häresie. Analog zu den Argumentationen Auers und Scheidings in diesem Band könnte man formulieren, dass in diesem Verständnis bereits in der Wahl der richtigen Materialien und Stoffe ebenso Mittel wie Ausdruck der Autopoiesis des Trachtlers liegen, hier zeigt und realisiert sich Zugehörigkeit zugleich.

33 Siehe zu diesem Zielkonflikt von Inklusivität und Profil auch den Beitrag von Scheiding in diesem Band.

34 Zur groben Orientierung: die Kosten für eine hochwertige, handgefertigte Tracht können leicht bei $€$ 3000,- liegen, Konfektionsware gibt es schon ab einem Zehntel dieser Summe. 
Schon für Gertrud Pesendorfer war die Frage nach den richtigen, guten und geeigneten Materialien eine zentrale. In ihrem früheren Band »Neue Deutsche Bauerntrachten« wettert sie gegen die »Überflutung und Übersättigung von seelenloser maschinell hergestellter Dutzendware " (Pesendorfer, I938, S. 7). Später erkennt sie zwar eine Balance aus »Handwebe « und »Fabrikware" an, immer verbunden jedoch mit einem deutlich sentimentalen Blick auf die wieder zu belebende gute alte Handwerkskunst: »Angefangen von der Arbeit auf dem Bauernhof, damit beginnend, daß die bäuerliche Heimarbeit, der bäuerliche Hausfleiß wieder angeregt wird und zu Ehren kommt [...] « (Pesendorfer, I966, S. XIII). Die Frage nach den geeigneten Materialien und Stoffen für die Tracht, im Sinne von Ästhetik, Haltbarkeit, Haptik und Verarbeitungsfähigkeit ist auch heute ein Dauerthema und Motiv, das verschiedene Felder und Szenerien unserer Feldforschungen durchzieht. Dies gilt sowohl für den ethnografierten Trachtennähkurs, bei dem die Teilnehmerinnen ob des gemeinsamen Zeigens, Betasten und Bestaunens der erworbenen Stoffe fast schon in eine Art kollektiven Qualitätsrausch gerieten, (vgl. Neuner-Schatz, 20I6, S. I26) als auch für eine Informationsveranstaltung der Südtiroler »Arbeitsgemeinschaft Lebendige Tracht «, bei der die Referentin ebenso ausführlich wie eindringlich die Bedeutung der Qualitätsfrage für die eigene Arbeit hervorhob. Man sei, im Gegensatz zu manchen Geschäftemachern, die sich leider auch im Bereich der Trachtenberatung tummelten, aber nur kommerzielle Interessen und das schnelle Geschäft mit billiger Massenware im Sinn hätten, nicht nur an qualitativ hochwertigen Trachten interessiert, sondern damit zugleich auch pflegerisch im Bereich des traditionellen Handwerks engagiert: »Die Arbeitsgemeinschaft setzt sich für die Förderung des heimischen Handwerks ein und spricht sich gegen eine fabriksmäßige Anfertigung aus. Ohne Handwerk keine Zukunft für die Tracht. «35

Über dieses grundsätzliche Bekenntnis zum »heimischen Handwerk « hinaus versteht sich diese Vereinigung jedoch auch als Multiplikatorin, Botschafterin und Pflegerin von traditionellem Wissen und traditionellen Fertigkeiten im Hinblick auf Materialwahl und Materialverarbeitung, welche in einer ganzen Reihe aus eigens herausgegeben Faltblättern zu Themen wie »Lederhose «, »Federkielstickerei «, »Zinngurt « und »Schneidertechnik « dargestellt werden. In all diesen Zusammenhängen wird die handwerklich alte und »richtige « Fertigungsweise erläutert, immer in Verbindung mit Verweisen auf Haltbarkeit (»fürs Leben«), Funktionalität, Echtheit und Schönheit der so erzeugten Stücke. Über die funktionalen Aspekte hinaus wird hier ein Bedeutungs- und Assoziationsfeld aufgerufen, das Gudrun König als »Manufactum-Prinzip»

35 www.hpv.bz.it/images/stories/arge-lebendige-tracht/folder-arge-lebendige-tracht.pdf, S. 2. (Zugriff: 21.04.20I7). 
bezeichnet hat: die Nobilitierung der Waren durch Geschichte und Geschichten, der Rückgriff auf Vergessenes und die Kombination aus konservatorisch-musealisierendem und reanimierendem Impuls (G.M. König, 20I 5, S. 279). Während sich in der bürgerlich-gediegenen Welt des Manufactum-Katalogs jedoch auch Momente der Ironie und des Augenzwinkerns identifizieren lassen (vgl. Bönisch-Brednich, 2002, S. I 59-I6I) ist es den hier vorgestellten Trachtlern sehr, sehr ernst: Es geht um weit mehr als Traditionspflege und Gemeinschaftserleben, das Geschehen ist von Untertönen der Modernisierungskritik samt Rettungsgedanken, der Authentizitätssuche und des Selbstentwurfs unterlegt: Nur in der echten Tracht ist der Trachtler echt.

\section{Fazit: Trachttragen als Selbstessentialisierung}

Ich komme zum Fazit und zugleich zur Ausgangsfrage zurück: Machen Trachten Menschenschläge? Jeggle und Korff fokussierten I974 in ihrem Beitrag über den Homo Zillertaliensis in einem groß angelegten, historisch gespannten Argumentationsbogen den Aspekt der konsequenten Internalisierung von erlernten Fremderwartungen unter den Bedingungen ökonomischer Notwendigkeit und des Tourismus: Die Show musste stimmen wie der Juchzger und sitzen wie der Trachtenjanker. Die Akteurinnen und Akteure erschienen wenn nicht als Opfer so doch als Resultate mächtiger historischer Dynamiken und externer ökonomischer Zwangsstrukturen, die sie auf Bühnen trieben und in Rollen zwängten. Der Blick in diesem Text richtet sich eher auf die internen Schauplätze jenseits touristisch präparierter Schauseiten. Hier zeigt sich ein vielschichtiges Feld des Ringens um Rang und Geltung. Wie Jeggle und Korff fragte ich danach, wie "ein Menschenschlag entsteht ", doch meine Antwort war kleinteiliger, situativer und performativer angelegt; sie weist den Akteurinnen selbst eine aktive Rolle zu. Die hier thematisierten Trachtler vollziehen in der Praxis des Trachttragens einen Akt der Selbstessentialisierung, indem sie semiotisch wie performativ eine sowohl zeitliche wie räumliche Verankerungsbehauptung auf sich als Person beziehen. Trachten sind wirksame Mittel der Kommunikation und der praktischen Herstellung von Differenzen und Differenzbehauptungen mit naturalisierendem Einschlag, da ihnen - hier habe ich mich von Auer (Abschnitt 2) in diesem Band inspirieren lassen - im geschilderten Sinn ein Moment der Verweigerung der Anerkennung von Kontingenz innewohnt.

Sowohl in der Abgrenzung zu Nicht-Trachtlern als auch in der feldinternen Positionierung hinsichtlich Ernsthaftigkeit und Qualitätsbewusstsein finden permanent Manöver von Zugehörigkeit und soziostruktureller Organisation statt. Die Tracht ist als Komplex sowohl 
materieller (Stoffe und Materialien) wie immaterieller (Wissensbestände und Klassifikationen) Bezüge und Komponenten ebenso Ausdruck wie Mittel multipler Abgrenzungs-, Differenzierungs- und Vergemeinschaftungsprozesse. Durch sie wird der »echte Trachtler « oder »die echte Trachtlerin " nach einer Kaskade an Distinktionen gegenüber den Nicht-Trachtlern, den nur »Trachtigen « oder den kommerziell oder industriell Verführten in einer Art Residualkategorie des Authentischen - je nach Perspektive - essentialisierend ontologisiert, kaserniert oder ausgestellt und damit zum Exemplar, Insassen oder Exponat gemacht. Menschenschläge gibt es nicht a priori - aber sehr wohl in der Selbstoder Fremdwahrnehmung. Und es gibt Mittel und Wege, ihre Existenz zu behaupten, z. B. aus Loden, Wollbrokat oder Hirschleder.

\section{Literatur}

Bodner, R. (2017): Das Leistungsabzeichen. Ein ethnografischer Erstkontakt mit volkskulturellen Dimensionen des Politischen. Unveröffentlichtes Manuskript, Innsbruck.

Bodner, R. \& Heimerdinger, T. (20I6): »Ein Erinnerungsfonds für die >Tiroler Volkskultur«? «. Österreichische Zeitschrift für Geschichtswissenschaften, 27(2), 168-208.

Bönisch-Brednich, B. (2002): »Der Manufactum-Katalog: Museale Objekte und Modernes Einkaufen ". Schweizerisches Archiv für Volkskunde, 98(I), I $5 \mathrm{I}-\mathrm{I} 65$.

Brückner, W. (I987): »Mode und Tracht. Ein Versuch «. In: K. Beitl \& O. Bockhorn (Hrsg.), Kleidung - Mode - Tracht: Referate der Österreichischen Volkskundetagung 1986 in Lienz (Osttirol) (S. I 5-43). Wien: Verein für Volkskunde.

Darnhofer, D. \& Rapp, I. (2015, Juni I4): »Goethe würde Augen machen «. Tiroler Tageszeitung Magazin, S. I-6.

Egger, S. (2008): Phänomen Wiesntracht. Identitätspraxen einer urbanen Gesellschaft. Dirndl und Lederhosen, München und das Oktoberfest. München: Utz.

Eggmann, S. \& Oehme-Jüngling, K. (2013): Doing society. >Volkskultur als gesellschaftliche Selbstverständigung. Basel: Schweizerische Gesellschaft für Volkskunde.

Glatzl, H. (unter dem K. gh) (I973): »Verwurzelte Tiroler und das Brauchtum ". Der Trachtler: Offizielles Organ des Tiroler Landestrachtenverbandes (S. I I-I2). Innsbruck: Tiroler Landestrachtenverband.

Goffman, E. (1986): »Techniken der Imagepflege«. In: Interaktionsrituale: Über Verhalten in direkter Kommunikation (I. Aufl, S. IO-54). Frankfurt a.M.: Suhrkamp. 
Grigo, J. (2015): Religiöse Kleidung: Vestimentäre Praxis zwischen Identität und Differenz. Bielefeld: Transcript.

Hansen, K. T. (2004): »The World in Dress: Anthropological Perspectives on Clothing, Fashion, and Culture «. Annual Review of Anthropology, 33, 369-392.

Hupfauf, S. (2016): Die Lieder der Geschwister Rainer und »Rainer Family aus dem Zillertal (I 822-1843): Untersuchungen zur Popularisierung von Tiroler Liedern in Deutschland, England und Amerika. (T. Nußbaumer, Hrsg.). Innsbruck: Universitätsverlag Wagner.

Jeggle, U. \& Korff, G. (I974a): »Homo Zillertaliensis oder Wie ein Menschenschlag entsteht «. Der Bürger im Staat, 24(3), I 82-I 88.

Jeggle, U. \& Korff, G. (1974b): »Zur Entwicklung des Zillertaler Regionalcharakters". Zeitschrift für Volkskunde, 70, 39-57.

Kammerhofer-Aggermann, U. (20I4): » eine reiche Auswahl der herrlichsten Volkskostüme und der schönsten Menschentypen «. Etappen der Entstehung unseres gegenwärtigen Begriffs von Tracht«. In: H. Justnik (Hrsg.), Gestellt: Fotografie als Werkzeng in der Habsburgermonarchie (S. 57-70). Wien: Löcker.

Keller-Drescher, L. (20I 5): » Tracht< als Denkstil. Zum Wissensmodus volkskundlicher Kleidungsforschung «. In: G.M. König, G. Mentges \& M.R. Müller (Hrsg.), Die Wissenschaften der Mode (S. I69-I 84). Bielefeld: Transcript.

König, A. (2007): Kleider schaffen Ordnung: Regeln und Mythen jugendlicher Selbst-Präsentation. Konstanz: UVK.

König, G.M. (2015): »Metamorphische Prozesse. Der kuratierte Konsum, das Sammeln und die Museumsobjekte«. Österreichische Zeitschrift für Volkskunde, LXIX/I I 8, 277-294.

Köstlin, E. (1999): Zur Wurzelmetaphorik in der Kulturwissenschaft. Eine Ikonographie (unveröffentlichte Dissertation). Graz.

Leimgruber, W. (200I): » Natürliche< und 'kulturelle< Faktoren bei der Konstruktion von Minderheiten. Das Beispiel der Jenischen«. In: R. W. Brednich (Hrsg.), Natur - Kultur: Volkskundliche Perspektiven auf Mensch und Umwelt; in Halle vom 27.9. bis I.10.1999 (S. 107I23). Münster New York München Berlin: Waxmann.

Marsel, M. (20I6): »Trachttragen zwischen Lust und Angst «. In: K. Eisch-Angus \& Erhard Löcker Ges.m.b.H (Hrsg.), Unheimlich heimisch: kulturwissenschaftliche BeTRACHTungen zur volkskundlich-musealen Inszenierung (S. I 57-168). Wien: Löcker.

Neuner-Schatz, N. (2016). Wissen Macht Tracht im Ötztal (Masterarbeit. Open access). Innsbruck. Abgerufen von http://diglib.uibk.ac.at/ ulbtirolhs/download/pdf/I77 I044? originalFilename=true

Pesendorfer, G. (1938): Tirol: Neue deutsche Bauerntrachten. München: Callwey. 
Pesendorfer, G. (1966): Lebendige Tracht in Tirol (2. Aufl.: I982). Innsbruck: Wagner.

Tiroler Landestrachtenverband. (20I4): Lernbehelf für volkskulturelles Leistungsabzeichen. Bronze. unveröffentlichtes Manuskript.

Wallnöfer, E. (2008): »Trachtenforschung als rassische Delimitation. Gertrud Pesendorfer (I895-I982), Gretl Karasek (I910-I992), Erna Piffl (1904-I987) «. In: E. Wallnöfer (Hrsg.), Maß nehmen, Maß halten: Frauen im Fach Volkskunde (S. 25-52). Wien Köln Weimar: Böhlau.

Wietschorke, J. (20I2, Juni I 5): »Historische Anthropologie und Europäische Ethnologie: Zur epistemologischen Verklammerung von Geschichte und Gegenwart in einem Forschungsprogramm «. H-SozKult. Kommunikation und Fachinformation für die Geschichtswissenschaften. Abgerufen I I. Mai 20I7, von http://www.hsozkult.de/ debate/id/diskussionen-I 799

Wietschorke, J. \& Schmidt-Lauber, B. (Hrsg.) (20I6). »Volkskultur 2.0«. Österreichische Zeitschrift für Geschichtswissenschaften, 27(2). 